\title{
EI foro de debate en línea en el aula de traducción
}

\section{Francisco Javier Fernández Polo y Mario Cal Varela}

Universidade de Santiago de Compostela

\begin{abstract}
In this paper we describe a teaching experience consisting in the use of online discussion forums as a complement of class sessions in a university course on translation. We discuss the main design features of the activity, specifically those referring to the major goals of the course, and comment on the results of its implementation throughout a semester. The paper ends with an assessment of the observed learning impact from both the point of view of the lecturing team and of the participating students.
\end{abstract}

Keywords: online discussion group, translation teaching, cooperative learning, higher education.

\begin{abstract}
Resumen
En esta comunicación describimos una experiencia docente consistente en la utilización de foros de discusión en línea como complemento de las clases presenciales en un curso de traducción de nivel universitario. Presentamos los aspectos esenciales del diseño de la actividad, en particular los referidos a los objetivos principales del curso, y comentamos su desarrollo a lo largo de un semestre. El trabajo concluye con una valoración del impacto observado sobre el aprendizaje tanto desde el punto de vista de los docentes de la materia como de los propios alumnos implicados.
\end{abstract}

Palabras clave: foro de debate en línea, enseñanza de la traducción, aprendizaje cooperativo, educación superior.

\section{Introducción}

Existe una amplia literatura sobre los beneficios de la comunicación asincrónica en línea para el aprendizaje. En este trabajo analizamos el desarrollo y resultados de una experiencia de aprendizaje cooperativo en línea en la enseñanza de la traducción en un contexto universitario, a través de foros de discusión en línea. El análisis describe la puesta en marcha y los resultados de la actividad en una asignatura obligatoria de segundo curso del Grado en Lengua y Literatura inglesas, y pone de relieve los beneficios, pero también los problemas surgidos, susceptibles de comprometer el valor pedagógico de la herramienta. 


\section{Objetivos}

Los objetivos de la iniciativa descrita en este trabajo concuerdan con los de otras experiencias de aprendizaje previas en la asignatura (Traducción directa e inversa (inglés)), tales como el uso de portafolios del alumno (Fernández Polo \& Cal Varela, 2011) o los proyectos de traducción en grupo para la Wikipedia. Los fines específicos que nos fijamos para esta actividad están íntimamente ligados a las ventajas pedagógicas asociadas a la utilización de la comunicación asincrónica en línea en contextos de educación superior (Garrison, 2003, Rourke et al., 1999) y a las propuestas más recientes en pedagogía de la traducción (Gile, 2009, González Davies, 2005, Hurtado Albir, 1996). Dichos objetivos son los siguientes:

a. Desplazar la atención de los alumnos del producto final de las tareas de traducción hacia el propio proceso de elaboración, de tal modo que se hagan explícitos los procesos de investigación y la toma de decisiones. El enfoque en el proceso es deseable en la primera etapa de la formación del traductor (Gile, 2009, 14), como es nuestro caso. Los foros permiten incorporar ambas perspectivas, el proceso y el producto: se discuten buenas y malas soluciones (producto), pero también cómo se ha llegado a ellas (posibles déficits en la lectura, selección y manejo de las fuentes de documentación, falta de atención a la revisión del texto, etc.).

b. Invitar a los estudiantes a reflexionar en profundidad sobre sus estrategias a la hora de identificar y resolver problemas de traducción. La reflexión sobre sus actividades "increases their self-confidence as translators and contributes to greater coherence, quality and speed in their translations" (González, 2005, 74). La reflexión constituye además una característica intrínseca a la comunicación asincrónica en línea (Biber \& Conrad, 2009, Garrison, 2003) y uno de sus principales beneficios para el aprendizaje.

c. Expandir el marco de aprendizaje más allá del aula hacia un ámbito en el que el papel del profesor ocupa un lugar menos central, de modo que los procesos de reflexión iniciados o solo apuntados en las clases presenciales se desarrollen de forma más libre y creativa. El foro de discusión parte de un enfoque del aprendizaje no "transmisionista", sino "transaccional” y "transformacional" (González Davies, 2004), basado en el aprendizaje cooperativo, que supone un mayor empoderamiento del alumno. Como apunta Garrison (2003, 56), "Lecturing online or simply providing access to information is a complete misuse of asynchronous learning networks", de ahí que el papel del profesor no debe ser tanto el de simple transmisor de información, como el de facilitador y moderador: "The asynchronous and virtual nature of online learning calls on learners to be self-directed and to take responsibility for their learning" (Garrison, 2003, 51).

d. Desarrollar las capacidades de los alumnos para el aprendizaje cooperativo, una competencia transversal de importancia capital tanto por su impacto sobre el propio aprendizaje como por sus implicaciones en relación con la capacitación para el futuro ejercicio profesional. Esto significa poder involucrar a los alumnos en una tarea de equipo en la que el objetivo final requiere de la colaboración de todos y en la que la 
diversidad de perspectivas e incluso las propuestas erróneas forman parte necesaria del proceso de construcción colectivo. La comunicación asincrónica en línea ofrece grandes ventajas para el aprendizaje cooperativo y la creación de una comunidad de aprendizaje (community of inquiry): "At the core of the properties of asynchronous online learning is the ability to provide collaborative learning experiences" (Garrison, 2003, 48).

e. Crear un clima favorable para el aprendizaje en grupo. Aprender a gestionar adecuadamente las críticas, tanto las que hacemos como las que recibimos, y aceptar que aprendemos constantemente de otros constituye un objetivo clave de esta propuesta formativa. Por otra parte, como el juego (González Davies, 2004, 4), el foro de discusión en línea puede proporcionar un ámbito de aprendizaje más relajado, desinhibidor y favorecedor del sentido de grupo.

f. Conectar a los alumnos con el mundo real profesional y superar el carácter artificioso del aula, familiarizándolos con herramientas de trabajo fundamentales en el mundo profesional de la traducción, en el que las nuevas tecnologías y la capacidad para trabajar en red en colaboración con otros profesionales resultan clave para el éxito en el desempeño profesional.

g. Fomentar la participación de los alumnos. Los foros en línea no sólo permiten implicar en el aprendizaje a los alumnos ausentes en el aula, sino también gestionar la diversidad, dando voz y aprovechando las aportaciones a) de los alumnos extranjeros, que por sus dificultades con el idioma suelen sentirse cohibidos a la hora de intervenir cara a cara en el aula, y b), en general, de los alumnos menos "rápidos" en la reflexión o que se estresan a la hora de expresar sus opiniones en público. Como apunta Susan Cain en una entrevista para Science of Us: "You very often find that the students who are more reticent to raise their hands are much more vocal when they're typing into an online forum" (Dahl, 2016).

\section{Desarrollo de la innovación}

\subsection{Contexto de la actividad}

La actividad forma parte de un curso de traducción general inglés-español-inglés, impartido mayoritariamente a alumnos de segundo curso del Grado en Lengua y Literatura Inglesas (Univ. de Santiago de Compostela). La duración es de un semestre (aproximadamente 14 semanas), con tres horas de clase presencial a la semana. Como complemento, el curso prevé la utilización del campus virtual a través de la plataforma Moodle. El número de estudiantes ronda los 150-200 e incluye un importante porcentaje de alumnos de intercambio, la mayoría con lenguas maternas distintas del español y el inglés. El profesorado es especialista en el tema y posee formación específica y experiencia en la utilización de plataformas de enseñanza virtual.

(c) EY-NC-ND 2016, Universitat Politècnica de València

Congreso In-Red (2016) 


\subsection{Desarrollo de la actividad}

La actividad, consistente en la utilización de foros en línea para la discusión de traducciones, se presenta en la sesión inicial del curso, insistiendo en sus objetivos y su peso en la evaluación final.

El foro en línea está concebido como un complemento al debate iniciado en la clase presencial en relación con un encargo semanal de traducción. El debate tiene por objeto la traducción de un extracto breve (100-150 palabras) del texto semanal. Generalmente, cada texto da lugar a dos o tres foros de discusión independientes en los que se discuten distintos fragmentos. La gestión de cada foro corre a cargo de un alumno voluntario, quien somete para el debate su propia propuesta de traducción y actúa como moderador de la actividad. La tarea se desarrolla en 5 fases:

1. mensaje inicial del profesor con instrucciones (texto, instrucciones básicas, plazos, nombre del moderador),

2. propuesta inicial del alumno-moderador,

3. comentarios de los demás participantes,

4. propuesta final y resumen-valoración del debate por parte del moderador, y

5. valoración y comentarios finales del profesor.

En línea con las propuestas de los especialistas en educación y nuevas tecnologías, el papel del docente en la actividad es el de facilitar, asesorar y guiar su desarrollo, en aras a convertirla en un instrumento de aprendizaje autónomo y grupal. Tal como sostiene Garrison $(2003,55)$ :

The most effective practice in establishing an online cognitive presence congruent with higher-order learning is for the teacher or facilitator to model reflective inquiry. This is best done with the teacher objectively providing commentary and insight into their thinking process (...) the teacher must participate in, but not dominate, discussions.

En este sentido, el rol del profesor se limita a la realización del encargo inicial, a evaluar las intervenciones de cada uno de los participantes (a través de una escala de 5 puntos, donde 1 es "útil" y 5 es "excelente") y a emitir un comentario-valoración final del foro. El comentario final tiene dos cometidos: a) destacar los aspectos más importantes del debate y extraer su valor pedagógico; y b) corregir errores tanto en la forma de las intervenciones como en las conclusiones del debate. El profesor explica al principio del curso, y en varias ocasiones a lo largo del mismo, cuáles son los factores que inciden en la calidad de las intervenciones para lograr que el foro sea un instrumento de aprendizaje eficaz, a saber: a) presentar argumentos con claridad y de manera ordenada; b) aportar pruebas que demuestren la validez de las opiniones expuestas; y c) tener en cuenta e incorporar en los comentarios las opiniones de los demás participantes. Estos factores son los principales criterios de calidad utilizados en la valoración de las intervenciones. 


\section{Resultados}

Los datos analizados se refieren al desarrollo de la experiencia en el curso académico 2014/15 (segundo semestre). El volumen de la actividad desarrollada a lo largo del semestre es el que queda recogido en la Tabla 1.

Tabla 1 Resumen de actividad a lo largo del semestre

\begin{tabular}{|l|l|}
\hline $\mathrm{N}^{\mathrm{o}}$ de textos & 7 \\
\hline $\mathrm{N}^{\mathrm{o}}$ de foros & 16 \\
\hline $\mathrm{N}^{\mathrm{o}}$ de posts & 1.061 \\
\hline $\mathrm{N}^{\mathrm{o}}$ total de palabras & $179.342(169$ palabras/mensaje $)$ \\
\hline
\end{tabular}

\subsection{Evolución de las intervenciones de los alumnos}

En general, tal y como se puede observar en la Figura 1, a lo largo del semestre se percibe un claro incremento a) en el número de participaciones en el foro y b) en el tamaño y complejidad de las mismas. No obstante esta tendencia, aunque no en el tamaño de las intervenciones, en general, se aprecia una pequeña disminución en el número de posts hacia el final del periodo, lo que concuerda con los resultados de otros trabajos (Swan, 2002), que indican un pico de actividad en torno al tercer cuarto y una ligera disminución en el último.

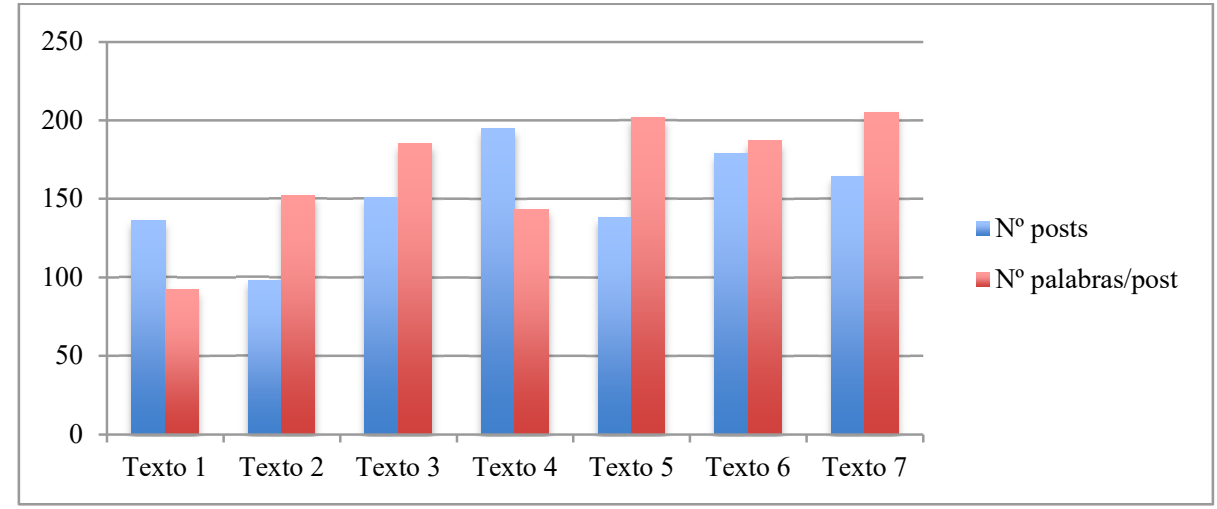

Fig. 1 Evolución de la participación a lo largo del semestre.

Los foros iniciales poseen un fuerte carácter monológico (se centran en el autor y en su punto de vista), se limitan a la expresión de la opinión del autor en referencia a la propuesta inicial del moderador y, en general, son escasas o inexistentes las referencias a otros participantes y al grupo. Con el tiempo, se observa una mayor conciencia del carácter dialógico del género, que gana en complejidad, apareciendo secciones nuevas, sobre todo de carácter interpersonal, plagadas de referencias a la audiencia (al moderador y al grupo). Se percibe, en este sentido, un incremento notable en la intensidad de los marcadores de "presencia social" (Rourke et al., 1999), como destaca Swan $(2002,26)$, "one of the 
cornerstones for the development of online learning communities" ${ }^{\text {: }}$ : saludos, vocativos, referencias al grupo, referencias (críticas y alabanzas) a las propuestas e intervenciones de otros participantes, etc. Se observa igualmente un importante aumento del tamaño de las secciones en las que se elaboran los argumentos y se explicitan las fuentes y, en general, las pruebas que apoyan las opiniones y propuestas del autor del post, tales como referencias a diccionarios o ejemplos de buenos usos encontrados en la red. A modo de ejemplo, presentamos aquí tres mensajes enviados por un mismo alumno en tres momentos distintos del semestre, que muestran las pautas observadas en las fases inicial, intermedia y final de la actividad. El subrayado indica aquellos aspectos más significativos en la evolución de las intervenciones:

Post 1

Yo traduciría "a glass of iced" como una "taza" y no "vaso", porque creo que queda mejor en el contexto. Reformularía la frase siguiente puesto que (...) También eliminaría los guiones, ya que en castellano en este caso se omitiría y $\underline{\text { se }}$ pondría una coma al final para aclarar el ",dijo".

\section{Post 4}

Hola!

En primer lugar, como bien han mencionado ya algunos de mis compañeros, yo tampoco optaría por (...) También estoy de acuerdo con Elena y no deberías (...) $\underline{\mathrm{Y} \text { como bien te han dicho, el equivalente a "dutch" es el idioma flamenco o }}$ neerlandés.

\section{Post 7}

Hola Xoel!

Por lo general, mi traducción es muy parecida a la tuya pero si tengo cosillas distintas.

(...) No disfrazas a una virgen sino que yo utilizaría un verbo tan simple como lo es "vestir" que como lo define la RAE "Cubrir o adornar el cuerpo con ropa" encaja muy bien en el contexto. (...)

$\underline{\text { Un saludo! }}$

En general, pues, el análisis de las intervenciones sugiere un comienzo vacilante de los participantes, para quienes la herramienta y la experiencia resulta probablemente nueva e ignoran cómo gestionarla adecuadamente. En las primeras fases de la actividad, puede verse como los participantes soslayan la necesidad de establecer vínculos interpersonales que ayuden a cubrir la distancia psicológica inherente a la comunicación en línea, un componente que, como destacamos anteriormente, Swan $(2002,42)$ considera clave para la creación de una comunidad de aprendizaje.

A medida que avanza el curso, se observa una progresiva incorporación en las intervenciones de marcadores de presencia social, tal como se puede ver en los ejemplos anteriores.

\footnotetext{
1 Social presence supports cognitive objectives through its ability to instigate, sustain, and support critical thinking in a community of learners. It supports affective objectives by making the group interactions appealing, engaging, and thus intrinsically rewarding, leading to an increase in academic, social, and institutional integration
} 
Con el paso del tiempo, las intervenciones se vuelven más ricas y ganan en valor pedagógico. En los primeros foros, el foco de atención se sitúa casi exclusivamente en el producto final, en la discusión sobre la traducción ideal. Sin embargo, paulatinamente se incorporan consideraciones referidas al proceso de traducción, tales como referencias a la calidad de las fuentes de documentación, el uso de herramientas de apoyo a la traducción (diccionarios y glosarios en línea), estrategias de búsqueda en la red, etc. En general, los foros ganan en reflexión y colaboración entre los participantes, dos puntos fuertes del aprendizaje en línea frente a las clases presenciales y que resultan clave para el aprendizaje efectivo, tal como señala Garrison (2003, 55): "recognizing and utilizing the unique capability of asynchronous learning networks to provide reflective and collaborative learning opportunities is the first step in enhancing learning effectiveness."

Lo que en definitiva vemos es un desarrollo de la competencia para la comunicación en línea, un proceso de adquisición de un nuevo género por parte de los alumnos y de optimización de su potencial para el aprendizaje. Paulatinamente los participantes en el foro van enriqueciendo sus intervenciones, en un proceso de aprendizaje colectivo en el que unos alumnos imitan pautas o modelos observados en otros compañeros, a la vez que incorporan las recomendaciones de los docentes en el comentario final.

\subsection{Valoración de la actividad}

\subsubsection{La perspectiva de los docentes}

Tras ya casi tres años de la introducción de esta actividad en el curso de traducción, la valoración que hacemos los docentes implicados es globalmente muy positiva. Los foros de discusión se han revelado como una herramienta eficaz para incentivar la participación activa de los estudiantes (vid. supra objetivo F). Durante el curso analizado, cada debate generó una media de 65 intervenciones, lo que supone un índice de participación muy superior al observado en las sesiones presenciales, en las que muchos alumnos suelen mostrarse poco proclives a dar su opinión y mucho menos a discutir la de otros compañeros, tal como recordaba Susan Cain en la entrevista citada más arriba.

La evolución cualitativa de las intervenciones descrita en la sección anterior es un claro indicio de que se ha producido un aprendizaje en el que no sólo han tenido que ver las indicaciones de los profesores sino también la interacción con los compañeros y la propia reflexión personal. La adquisición de autonomía y capacidad para trabajar sin la supervisión estrecha del docente (objetivos C y D) se ve confirmada en el proyecto de grupo que suele poner punto final al curso, $\mathrm{y}$ en el que la responsabilidad de los estudiantes es prácticamente total en las distintas fases del trabajo. En este sentido, cabe atribuir el alto nivel de los proyectos observado estos dos últimos años al aprendizaje previo a través de los foros.

No obstante lo dicho, el debate semanal en línea se ha revelado también como una herramienta bastante eficaz para valorar el alcance de ciertas disfunciones observadas en la dinámica habitual de las clases. Año tras año, la actividad pone de manifiesto la inexistencia de una verdadera cultura de la reflexión en un sistema que parece mostrar una

(c) EY-NC-ND 2016, Universitat Politècnica de València

Congreso In-Red (2016) 
atención casi exclusiva al resultado final de cualquier tarea y a la vez un enorme desdén por los errores y pasos en falso que necesariamente van señalando el camino. Es necesario un esfuerzo docente considerable - feedback constante de los docentes en las clases presenciales y en las intervenciones que cierran cada foro- para vencer esa inercia y provocar un cambio significativo de actitud en los estudiantes (objetivos A y B).

Como ya se ha apuntado, sobre todo en los debates iniciales del curso, los participantes tienden a desaprovechar las posibilidades interactivas del foro, establecen en muy contadas ocasiones un diálogo a múltiples bandas y a menudo "se descuelgan" de la discusión una vez realizada una única aportación. Su resistencia a "cambiar el chip" se observa además en rutinas un tanto mecánicas de interacción con el fin de cubrir expediente asumiendo pocos riesgos, aportando escaso contenido y mostrando poca o ninguna implicación personal con el grupo, más allá de algunas expresiones de cortesía muy convencionales.

Por otro lado, por su carácter intrínsecamente dialéctico, la actividad constituye un interesante marco en el que se ponen en juego afinidades y tensiones entre compañeros que se manifiestan de forma más o menos sutil en el desarrollo de los debates. La adecuada gestión de esta dimensión interpersonal es una faceta también esencial en el desarrollo de aptitudes para el trabajo en equipo (objetivo E).

Al cabo de casi tres años de experiencia con la puesta en práctica de esta actividad, nuestra valoración es globalmente muy positiva. La dificultad de su implementación estriba sin duda en hallar el adecuado nivel de "presencia docente" (Rourke \& al 1999), que actúe de catalizador sin limitar de forma destructiva el desarrollo autónomo de la actividad. La evolución de las intervenciones de los alumnos en los foros indica que las valoraciones cuantitativas y cualitativas de cada foro por parte de los docentes tienen un efecto apreciable, incluso en el estrecho marco temporal de un semestre.

\subsubsection{La perspectiva de los estudiantes}

Para conocer el punto de vista de los estudiantes sobre la actividad, elaboramos un cuestionario específico que aplicamos a través de la propia plataforma Moodle al término del curso académico 2014-2015. Un total de 71 participantes en ese curso completó la encuesta, cuyos resultados se resumen en la Figura 2. Además de los aspectos que se recogen en este gráfico ordenados de mejor a peor valorados, se les pidió a los alumnos que evaluasen globalmente la actividad. La puntuación media fue de 3,4 en una escala de cinco puntos en la que 1 era la valoración más negativa y 5 constituía una calificación de excelente. En conjunto, cabe afirmar que la reacción de los estudiantes ha sido bastante positiva, pero conviene no perder de vista su opinión sobre los distintos aspectos considerados individualmente. 


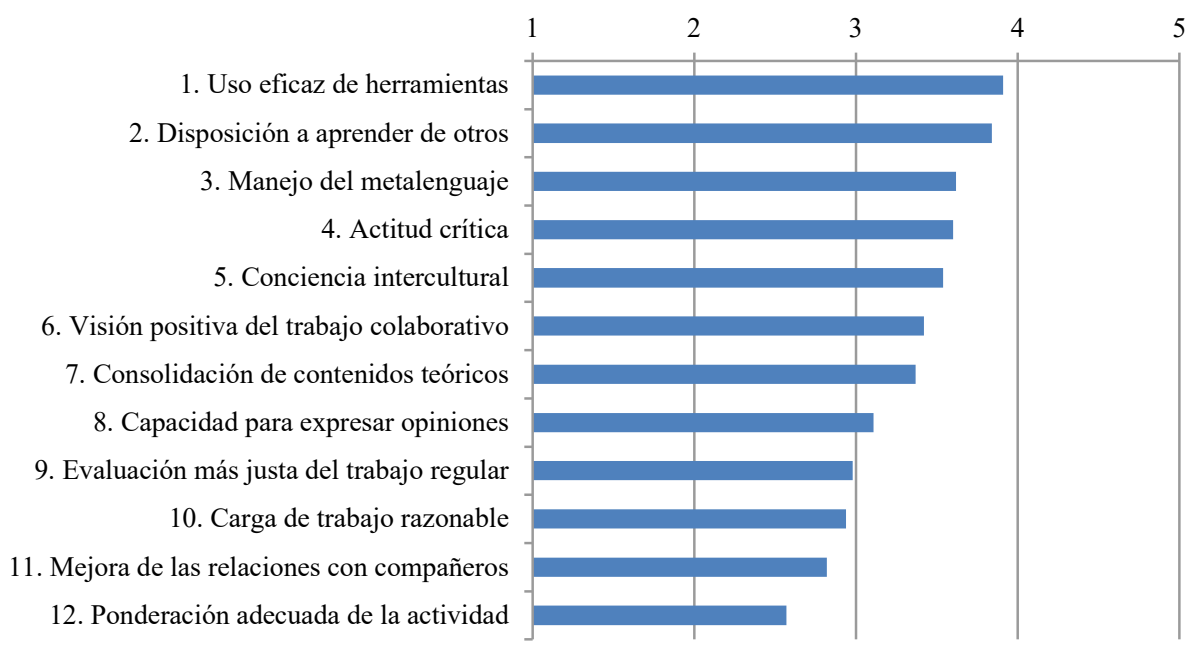

Fig. 2 Valoración del impacto positivo de la actividad sobre el aprendizaje y la evaluación.

Resulta alentador observar la posición destacada que ocupan varias dimensiones estrechamente relacionadas con los objetivos principales del curso. Como cabía esperar, la necesidad de aportar pruebas y justificar las opiniones ha tenido un efecto muy positivo sobre la eficacia y la confianza con la que los estudiantes manejan las herramientas y obras de referencia y el metalenguaje necesario para discutir con precisión sobre cuestiones lingüísticas complejas (objetivos A y B). Con una valoración global de 3,8, el efecto positivo sobre la predisposición a aprender de otros confirma el carácter innovador de la actividad, en la que el papel del profesor cede espacio a la responsabilidad de los propios alumnos en el aprendizaje (objetivos C y D). El aumento del espíritu crítico, cuarta dimensión mejor valorada, apunta también en esta dirección y constituye un indicio de que los alumnos han interiorizado los objetivos del curso y han hecho suyos los criterios de calidad adecuados para enfrentarse a la actividad de traducir. Por último, en este bloque de dimensiones valoradas por encima de 3,5 se encuentra también el desarrollo de la conciencia intercultural. El carácter escrito y el ritmo más reposado de la discusión asincrónica sin duda facilitan la integración de las distintas voces y permiten explotar en mayor medida la heterogeneidad cultural del aula (objetivo G).

Un segundo bloque de 3 ítems del cuestionario se sitúa entre 3 y 3,5 puntos, lo que constituye una valoración relativamente positiva de los aspectos considerados. Para un número significativo de estudiantes, la participación semanal en los foros en línea mejora su visión personal del trabajo colaborativo y su capacidad para expresar y defender opiniones con claridad ante el resto de la clase (objetivo E), aspectos ambos que repercuten de forma positiva en el desarrollo de dinámicas más participativas en las clases presenciales. Los estudiantes perciben además que el debate en línea contribuye a consolidar los contenidos expuestos y discutidos en las clases.

(c) EY-NC-ND 2016, Universitat Politècnica de València

Congreso In-Red (2016) 
Quedan para el final cuatro aspectos cuya valoración inferior a 3 invita a la reflexión y quizás exige un replanteamiento de ciertos aspectos de la actividad. Tres de estas dimensiones están en realidad relacionadas con la evaluación y, en cierto modo, podrían tener una lectura positiva. Los alumnos sienten que el tiempo y el esfuerzo invertidos en la tarea no se ven adecuadamente recogidos en la calificación obtenida, lo que, hasta cierto punto, cabría interpretar como que valoran la actividad por encima de lo que refleja su impacto en la nota final. Por otra parte, a la luz de los comentarios que aportan en las secciones abiertas del cuestionario, parece evidente que el hecho de que se trate de un ejercicio evaluado limita en exceso la espontaneidad de las intervenciones y condiciona más de lo esperado el desarrollo de la actividad. En este sentido, a juzgar por los resultados de la encuesta, muchos consideran que los foros de discusión no contribuyen positivamente a mejorar las relaciones con los compañeros. En concreto, la evaluación cuantitativa individualizada de las intervenciones por parte del docente pretendía incentivar una mayor elaboración y mayor profundidad en las reflexiones, objetivo que parece haberse cumplido al menos en parte, a juzgar por la evolución de las intervenciones en los foros descrita anteriormente. Sin embargo, para un sector del alumnado, esa evaluación generó cierto nivel de ansiedad y de competitividad entre los participantes que puede haber disuadido a muchos de participar con regularidad en los foros.

\section{Conclusiones}

En esta descripción de nuestra experiencia con la utilización de foros de discusión en línea en el aula de traducción hemos tratado de pasar revista a aquellas potencialidades de esta herramienta que la convierten en un valioso instrumento para fomentar la colaboración entre alumnos y el aprendizaje en grupo. Hemos procurado ofrecer una visión desde distintos ángulos para identificar también algunas disfunciones que con seguridad habrán limitado sus beneficios en un grado todavía difícil de determinar.

Con respecto a los objetivos marcados inicialmente, los resultados observados los dos últimos cursos pueden calificarse de muy positivos. La participación ha sido razonablemente amplia y sostenida en el tiempo y ha reflejado de forma bastante adecuada la diversidad cultural del aula. El carácter asincrónico de los foros favorece la reflexión pausada y profunda sobre el proceso de aprendizaje y facilita la posibilidad de compartir ideas y aprender de los compañeros. En nuestra experiencia, el debate en línea se revela como un medio doblemente útil para romper con dinámicas de clase demasiado tradicionales: por un lado, releva al profesor de su responsabilidad como fuente casi única de aprendizaje y, por el otro, desplaza el centro de atención del resultado final de una tarea y lo coloca en el propio proceso de elaboración. En el foro, los alumnos explicitan y discuten diversas soluciones posibles a un mismo problema de traducción y ensayan criterios y estrategias, poniendo con ello a prueba y desarrollando su espíritu crítico. El propio diseño de la actividad, en la que los estudiantes ayudan al compañero moderador a mejorar su propuesta inicial, favorece el sentido de equipo de la clase, en tanto que comunidad de aprendizaje. 
Es quizás en esta última faceta de la actividad donde las opiniones de los alumnos apuntan a la necesidad de introducir cambios, de tal modo que la evaluación de la actividad no interfiera negativamente en la creación de un clima de cooperación adecuado. El reto para el docente es saber conjugar la necesidad de ofrecerle a los alumnos un feedback crítico y formativo y al mismo tiempo favorecer un clima de participación razonablemente distendido que los anime a participar.

\section{Referencias}

BiBer, D., \& CONRAD, S. (2009). Register, genre, and style. Cambridge: Cambridge University Press. DAHL, M. (2016). How Schools Are Failing Their Quietest Students. Retrieved March 18, 2016, from http://nymag.com/scienceofus/2016/03/how-schools-are-failing-their-quietest-students.html

FERNÁNDEZ Polo, F. J., \& CAL VARELA, M. (2011). Learning translation through the use of portfolios: description of an experience. @tic. Revista D'innovació Educativa, 7, 44-51.

GARRISON, D. R. (2003). Cognitive presence for effective asynchronous online learning: the role of reflective inquiry, self-direction and metacognition. In J. Bourne \& J. C. Moore (Eds.), Elements of Quality Online Education: Practice and Direction (pp. 47-58). Needhamn: Sloan Center for Online Education.

GILE, D. (2009). Basic concepts and models for interpreter and translator training. Amsterdam: John Benjamins.

GonZÁlez DAVIES, M. (2004). Multiple voices in the translation classroom: activities, tasks and projects. Amsterdam: John Benjamins.

GONZÁLEZ DAVIES, M. (2005). Minding the process, improving the product: Alternatives to traditional translator training. In M. Tennent (Ed.), Training for the new millennium: pedagogies for translation and interpreting (pp. 67-82). Amsterdam: John Benjamins.

Hurtado Albir, A. (Ed.). (1996). La enseñanza de la traducción. Castelló de la Plana: Publicacións de la Universitat Jaume I.

Rourke, L., Anderson, T., Garrison, D. R., \& Archer, W. (1999). Assessing Social Presence In Asynchronous Text-based Computer Conferencing. Journal of Distance Education, 14(2), 50 71.

SwAN, K. (2002). Building learning communities in online courses: The importance of interaction. Education, Communication \& Information, 2(1), 23-49.

(c) EY-NC-ND 2016, Universitat Politècnica de València

Congreso In-Red (2016) 